\title{
Résumé de la mise à jour du CCNI sur les vaccins contre le zona
}

\author{
$\mathrm{R}$ Warrington ${ }^{1,2}, \mathrm{~S}$ Ismail ${ }^{3}$ au nom du Comité consultatif national de l'immunisation (CCNI)*
}

\section{Résumé}

Contexte : Des augmentations abruptes de l'incidence du zona, du taux d'hospitalisations liées au zona et du risque de névralgies post-zostériennes consécutives au zona se produisent chez les personnes de plus de 50 ans. À l'heure actuelle, deux vaccins contre le zona sont homologués pour l'usage chez les personnes de 50 ans et plus au Canada : un vaccin à virus vivant atténué contre le zona (VVCZ), homologué en 2008, et un vaccin sous-unitaire recombinant (VRZ), homologué en octobre 2017.

Objectifs : Passer en revue les données probantes existantes et formuler des lignes directrices pour déterminer si le VVVCZ auparavant homologué (Zostavax ${ }^{\mathrm{MD}}$ ) et (ou) le VRZ qui a été homologué récemment (Shingrix ${ }^{\mathrm{MD}}$ ) devrait être offert aux Canadiennes et aux Canadiens âgés de 50 ans et plus : 1) à l'échelle de la population, dans le cadre de programmes de vaccination financés publiquement; et 2) à l'échelle individuelle, aux personnes qui souhaitent prévenir le zona, ou aux cliniciens qui désirent conseiller leurs patients au sujet de la prévention du zona.

Méthodologie : Le groupe de travail sur le zona du Comité consultatif national de I'immunisation (CCNI) a mis au point une stratégie de recherche prédéfinie afin de repérer toutes les études admissibles, a évalué la qualité de celles-ci, a analysé les données et a résumé ses observations. Une analyse coût-utilité du WVVCZ et du VRZ a également été effectuée à travers le point de vue du système de santé. Des recommandations ont finalement été formulées, conformément au processus fondé sur des données probantes du CCNI. La force des recommandations et de la qualité des données probantes sur lesquelles elles s'appuient ont été définies. Le CCNI a évalué et approuvé les recommandations à la lumière de ces données probantes.

Résultats : Cinq recommandations ont été formulées à l'appui de la prise de décisions à l'échelle de la santé publique et à l'échelle individuelle : 1) On devrait offrir le VRZ aux populations et aux personnes de 50 ans et plus qui ne présentent pas de contre-indication (forte recommandation du CCNI, données probantes de catégorie A). 2) On devrait offrir le VRZ aux populations et aux personnes de 50 ans et plus qui ont par le passé reçu le VVCZ et qui ne présentent pas de contre-indication (forte recommandation du CCNI, données probantes de catégorie A). La revaccination par I'administration de deux doses du VRZ peut être envisagée au moins un an après l'administration du VWVCZ (recommandation discrétionnaire du CCNI, données probantes de catégorie I). 3) On devrait offrir le VRZ aux populations et aux personnes de 50 ans et plus qui ne présentent pas de contre-indication et qui ont des antécédents de zona (forte recommandation du CCNI, données probantes de catégorie B). L'administration des deux doses du VRZ peut être envisagée au moins un an après le dernier épisode de zona (recommandation discrétionnaire du CCNI, données probantes de catégorie I). 4) On peut envisager l'administration du WVCZ aux populations et aux personnes de 50 ans et plus qui sont immunocompétentes et qui ne présentent pas de contre-indication, dans les cas où le VRZ est contre-indiqué, s'il est impossible de l'obtenir ou lorsqu'il est trop cher (recommandation discrétionnaire du CCNI, données probantes de catégorie A). 5) On peut envisager, au cas par cas, l'administration du VRZ (et non du VWCZ) chez les adultes immunodéprimés de 50 ans et plus (recommandation discrétionnaire du CCNI, données probantes de catégorie I).

Conclusion : Les deux vaccins sont immunogènes, sûrs et réduisent l'incidence du zona et de la névralgie post-zostérienne. L'efficacité du WVCZ contre le zona s'atténue en fonction de l'âge du récipiendaire et du temps écoulé depuis la vaccination. L'efficacité du VRZ demeure élevée et semble diminuer plus lentement que celle du VVVCZ, et ce, dans tous les groupes d'âge. Comparativement à l'absence de vaccination, les deux vaccins sont rentables chez les personnes de 50 ans et plus, et en particulier chez les 65 à 79 ans. Le VRZ est plus rentable que le WVCZ.

\author{
Affiliations \\ ${ }^{1}$ Président du groupe de travail \\ sur le zona du CCNI, Edmonton \\ (Alberta) \\ 2 Département de médecine \\ interne, Allergie et immunologie \\ clinique chez l'adulte, Université \\ du Manitoba, Winnipeg \\ (Manitoba) \\ ${ }^{3}$ Agence de la santé publique du \\ Canada, Centre de l'immunisation \\ et des maladies respiratoires \\ infectieuses, Ottawa (Ontario)
}

*Correspondance : phac.naciccni.aspc@canada.ca
Citation proposée : Warrington R, Ismail S. Résumé de la mise à jour du CCNI sur les vaccins contre le zona. Relevé des maladies transmissibles au Canada 2018;44(9):249-55. https://doi.org/10.14745/ccdr.v44i09a06f 


\section{Introduction}

Le zona est caractérisé par une douleur neuropathique et une éruption vésiculaire cutanée suivant la trajectoire d'un dermatome. La maladie est causée par la réactivation du virus zona-varicelle (VZV), qui survient en raison de l'atténuation de la réponse immunitaire cellulaire associée au vieillissement ou à une immunosuppression. La complication la plus fréquente du zona est la névralgie post-zostérienne, laquelle est souvent débilitante. Près d'un Canadien sur trois développera le zona au cours de sa vie (1). L'âge est le principal facteur de risque pour le zona, la névralgie post-zostérienne et les hospitalisations liées au zona, dont les incidences augmentent drastiquement après l'âge de 50 ans (2-7). Les taux les plus élevés d'hospitalisations dues au zona et à la névralgie post-zostérienne sont observés chez les 65 ans et plus $(1,4,7-9)$.

En 2008, un Vaccin à virus vivant atténué contre le zona (VVVCZ, Zostavax ${ }^{\mathrm{MD}}$ ) a été homologué au Canada pour utilisation chez les personnes de 60 ans. En 2011, son utilisation a été homologuée chez les personnes de 50 ans et plus. En 2010 et en 2014, le Comité consultatif national de l'immunisation (CCNI) a publié des recommandations fondées sur des données probantes concernant l'utilisation du WVCZ chez les personnes immunocompétentes de 60 ans et plus $(10,11)$. Le CCNI a également recommandé que le VVVCZ puisse être utilisé chez les patients de 50 à 59 ans car, bien que ce vaccin soit à la fois sûr et efficace pour ce groupe d'âge, la durée de la protection du vaccin était inconnue au-delà des cinq premières années et on ignorait si la vaccination procurerait une protection jusqu'à un âge avancé où le fardeau du zona devient plus important.

En mai 2014, le Comité canadien sur l'immunisation a recommandé d'offrir le VVVCZ de façon systématique aux adultes immunocompétents âgés de 60 à 65 ans ne présentant aucune contre-indication. Cette recommandation repose sur l'épidémiologie du zona, sur les caractéristiques du vaccin contre le zona, sur la modélisation de la maladie et les analyses économiques, ainsi que sur la faisabilité et l'acceptabilité des programmes d'immunisation contre le zona (12). Bien que le WVCZ ait été offert pour la vente privée, aucun programme de vaccination financé par l'État n'était offert au Canada avant que l'Ontario n'offre le vaccin aux personnes de 65 à 70 ans en septembre 2016 (3).

En octobre 2017, le Canada était le premier pays à homologuer I'utilisation d'un vaccin sous-unitaire recombinant contre le zona (VRZ, Shingrix ${ }^{\mathrm{MD}}$ ) contenant la glycoprotéine $\mathrm{E}$ du VZV et le nouveau système adjuvant $A S 01_{B}$. Cette homologation a rendu nécessaire la publication d'une mise à jour de la déclaration du comité consultatif du CCNI concernant l'utilisation des vaccins contre le zona. L'objectif principal de cette déclaration est de passer en revue les données probantes existantes et de formuler des lignes directrices sur l'utilisation du vaccin sous-unitaire recombinant. Les lignes directrices indiquent aussi si le WVCZ (auparavant homologué) et (ou) le VRZ devrait être offert aux Canadiens de 50 ans et plus à l'échelle de la population (dans le cadre de programmes de vaccination financés publiquement) ainsi qu'à l'échelle individuelle (aux personnes souhaitant prévenir le zona et aux cliniciens désirant conseiller leurs patients au sujet de la prévention du zona au moyen de vaccins qui ne font pas partie des programmes de vaccination financés publiquement à l'heure actuelle). Tous les détails se trouvent dans la Mise à jour sur l'utilisation du vaccin contre le zona par le Comité consultatif national de l'immunisation (14). L'objectif du présent article est de résumer les principaux constats de la mise à jour.

\section{Méthodologie}

Le groupe de travail sur le zona du CCNI a effectué des revues de la littérature portant sur le zona et les vaccins contre le zona, et a examiné les données fournies par les fabricants de vaccins. Les données probantes ont été catégorisées, évaluées de manière critique et rapportées dans des tableaux. Des études portant sur l'immunogénicité, l'innocuité et l'efficacité du VRZ administré selon différents schémas posologiques à divers groupes de patients immunodéprimés âgés de 18 ans et plus étaient toujours en cours au moment des délibérations du CCNI; par conséquent, elles n'ont pas été incluses dans la présente revue.

Le CCNI surveillera les données probantes émergeantes portant sur l'administration des vaccins contre le zona chez les personnes immunodéprimées et en présentera une revue dans une autre déclaration du comité consultatif.

Une synthèse des connaissances a été réalisée, les données ont été évaluées de manière critique et le groupe de travail sur le zona a soumis des recommandations fondées sur des données probantes conformément au processus du CCNI (15). Ce processus comprenait la clarification du raisonnement et des considérations pertinentes. Une nouvelle terminologie a récemment été mise au point afin de définir la force des recommandations du CCNI :

- Une forte recommandation s'applique à la plupart des populations ou des personnes et devrait être suivie, à moins qu'il n'existe une justification claire et convaincante d'utiliser une approche alternative.

- Une recommandation discrétionnaire peut être envisagée pour certaines populations ou certaines personnes dans certaines circonstances. D'autres approches peuvent être acceptables.

\section{Résultats}

Le WVCZ ainsi que le VRZ ont montré qu'ils sont à la fois immunogènes et sûrs et efficaces pour réduire l'incidence du zona et de ses complications (telles que la névralgie post-zostérienne). L'efficacité du VVVCZ contre le zona diminue avec l'âge au moment de la vaccination et le temps écoulé depuis la vaccination. L'efficacité du VRZ demeure élevée et semble diminuer plus lentement que celle du VVVCZ, et ce, dans tous les groupes d'âge. Dans les trois ans suivant la vaccination, I'efficacité du VRZ contre l'incidence du zona et de la névralgie post-zostérienne semble être le double de celle du VVVCZ, duquel une diminution importante de la protection fut observée un an après son administration. En revanche, l'efficacité du VRZ contre l'incidence du zona demeure constante dans les quatre ans suivant la vaccination et aucune diminution significative n'a été observée avec le temps. Le VVVCZ est significativement moins efficace chez les adultes de plus de 70 ans, comparativement aux adultes de 50 à 59 ans, alors que l'efficacité du VRZ sur quatre ans ne présente aucune différence significative entre les groupes $d$ 'âge. Le VRZ est plus réactogène que le VVVCZ en raison de son adjuvant, qui provoque une forte réponse de l'immunité cellulaire afin d'aider à compenser la baisse de l'immunité liée au vieillissement. Comparativement à l'absence de vaccination, les deux vaccins sont rentables chez les 50 ans et plus, et en particulier chez les 65 à 79 ans; toutefois, du point de vue du système de santé, le VRZ est plus rentable que le VVVCZ. La revue de la littérature portant sur l'utilisation des vaccins contre le zona ainsi que les recommandations 
actuelles en matière de vaccination contre le zona sont publiées dans la version complète de la mise à jour de la déclaration du CCNI (14) et dans le chapitre sur le zona du Guide canadien d'immunisation (16).

\section{Recommandations et raisonnements}

En s'appuyant sur les raisonnements suivants, le CCNI a approuvé cinq recommandations pour la prise de décisions à l'échelle de la population et à l'échelle individuelle.

1. On devrait offrir le vaccin sous-unitaire recombinant aux populations et aux personnes de 50 ans et plus qui ne présentent pas de contre-indication (forte recommandation du $\mathrm{CCNI}$, données probantes de catégorie A).

Le VVCZ et le VRZ sont à la fois immunogènes et sûrs et efficaces contre le zona et la névralgie post-zostérienne. Après réflexion, le CCNI a jugé que l'efficacité supérieure du VRZ chez les adultes de 50 ans et plus, l'amenuisement négligeable de la protection qu'il procure et sa rentabilité appuient une recommandation destinée aux programmes de santé publique et favorisant la vaccination des populations de 50 ans et plus, qui sont exposées à un risque accru de zona et de névralgie post-zostérienne et à qui le VRZ procurera probablement une protection continue à un âge plus avancé, alors que le risque de zona et de névralgie post-zostérienne continue d'augmenter. À l'échelle des programmes de santé publique, le VRZ s'est montré plus rentable que le VVVCZ. Ces programmes auront besoin de stratégies ( $p$. ex. éducation, rappels) visant à assurer l'adhésion au schéma posologique en deux doses du VRZ (étant donné que la durée de la protection suivant une dose unique n'est pas connue) et à offrir des conseils relatifs à la réactogénicité à court terme du vaccin. Si, en raison de contraintes opérationnelles, il faut définir les priorités des programmes ciblés de vaccination, les provinces et les territoires pourraient tenir compte des avantages relatifs (sur les plans de l'épidémiologie et de la rentabilité) de la vaccination de cohortes de différents groupes d'âge. À l'échelle individuelle, les personnes souhaitant prévenir le zona et les cliniciens désirant conseiller leurs patients au sujet de la prévention du zona peuvent tenir compte du prix des vaccins. Les individus doivent être prêts à respecter le schéma posologique en deux doses du VRZ (étant donné que la durée de la protection suivant une dose unique n'est pas connue) et comprendre qu'ils pourraient s'exposer à la réactogénicité à court terme de ce vaccin.

2. On devrait offrir le VRZ aux populations et aux personnes de 50 ans et plus qui ont déjà reçu le VVVCZ et qui ne présentent pas de contre-indication (forte recommandation du $\mathrm{CCNI}$, données probantes de catégorie A).

2a. La revaccination par l'administration de deux doses du VRZ peut être envisagée un an après l'administration du VVVCZ (recommandation discrétionnaire du CCNI, données probantes de catégorie I).

Les personnes ayant déjà reçu le VVVCZ recevront une meilleure protection avec l'administration des deux doses du VRZ, compte tenu de l'efficacité accrue et persistante de ce dernier dans tous les groupes d'âge. L'innocuité, la réactogénicité et l'immunogénicité du VRZ administré aux personnes qui avaient déjà reçu le VVVCZ se sont montrées comparables à ce qui a été observé lorsque le VRZ a été administré aux personnes n'ayant jamais reçu le VVVCZ. Chez les personnes qui ont déjà reçu le WVVCZ, le délai avant l'administration du VRZ dépendra de l'âge au moment de l'administration du VVVCZ (étant donné que l'efficacité du vaccin diminue avec l'âge) et du temps écoulé depuis la vaccination (étant donné que l'efficacité du vaccin diminue après la première année). En s'appuyant sur des données probantes limitées, le CCNI propose la vaccination par I'administration de deux doses du VRZ au moins un an après I'administration du WVVCZ, en raison de la baisse rapide de l'efficacité de ce dernier après un an. Bien que l'intervalle utilisé dans la seule étude ayant porté sur une telle revaccination à avoir été publiée ait été de cinq ans, il n'y a aucune raison de croire qu'un intervalle plus court pourrait être nuisible.

3. On devrait offrir le VRZ aux populations et aux personnes de $\mathbf{5 0}$ ans et plus qui ont des antécédents de zona et qui ne présentent pas de contre-indication (forte recommandation du $\mathrm{CCNI}$, données probantes de catégorie A).

3a. L'administration des deux doses du VRZ peut être envisagée au moins un an après l'épisode de zona (recommandation discrétionnaire du CCNI, données probantes de catégorie I).

Comme il I'avait fait dans ses recommandations de 2014, le CCNI recommande l'administration du VRZ chez les personnes qui ont déjà eu un épisode de zona. Ces personnes continuent d'être exposées au risque de développer le zona et il est impossible de se fier aux antécédents de zona pour prédire de nouveaux épisodes. Par conséquent, I'administration du VRZ serait bénéfique pour les personnes qui rapportent des antécédents de zona. Par ailleurs, une étude a démontré que l'administration du VRZ au sein de cette population n'entraînait aucune différence sur les plans de l'innocuité et de l'immunogénicité. En l'absence de données probantes indiquant le délai approprié avant l'administration du vaccin, le CCNI maintient sa proposition antérieure, selon laquelle il est recommandé d'attendre au moins un an après l'épisode de zona avant $d$ 'administrer le vaccin contre le zona.

4. Il est possible d'envisager l'administration du VVVCZ aux populations et aux personnes de 50 ans et plus qui sont immunocompétentes et qui ne présentent pas de contre-indication, dans les cas où le VRZ est contre-indiqué, s'il est impossible de l'obtenir ou lorsqu'il est trop cher (recommandation discrétionnaire du CCNI, données probantes de catégorie A).

Le CCNI conclut (comme il l'avait fait dans les déclarations antérieures du comité consultatif) qu'il existe des données probantes suffisantes pour recommander l'administration du WVCZ chez les adultes de 60 ans et plus (données probantes de catégorie A). Toutefois, la recommandation sur l'utilisation de ce vaccin chez les populations immunocompétentes de 60 ans et plus est dorénavant « discrétionnaire » en raison des données comparatives démontrant l'efficacité supérieure, la durée prolongée de la protection et la rentabilité relative du VRZ qui a récemment été homologué. Bien que le VVVCZ soit efficace et sûr chez les personnes de 50 à 59 ans et que le CCNI I'ait déjà recommandé sur une base discrétionnaire dans ce groupe d'âge, la diminution de la protection de ce vaccin fait en sorte qu'il ne pourrait possiblement pas procurer une protection continue optimale chez les personnes plus âgées, lorsque le risque de zona et de névralgie post-zostérienne devient le plus élevé. Avec l'homologation récente du VRZ, qui présente une efficacité supérieure et une durée de protection prolongée dans ce groupe d'âge, le CCNI recommande fortement que ce vaccin soit désormais utilisé chez les adultes de 50 à 59 ans comme chez les adultes de 60 ans et plus qui ne présentent aucune contre-indication. L'administration du VVVCZ peut toujours être envisagée dans les cas où le VRZ est contre-indiqué (c.-à-d. 
hypersensibilité connue à tout composant du vaccin), s'il est impossible d'obtenir le VRZ ou si son prix le rend inaccessible. Le WVCZ est homologué au Canada depuis 2008. II s'est montré immunogène, efficace et sûr.

5. Il est possible d'envisager, au cas par cas, I'administration du VRZ (et non du VVVCZ) chez les adultes immunodéprimés de 50 ans et plus (recommandation discrétionnaire du $\mathrm{CCNI}$, données probantes de catégorie I).

Contrairement au VVVCZ, I'immunosuppression n'est pas une contre-indication au VRZ. Compte tenu du fardeau du zona chez les personnes immunodéprimées ainsi que des lignes directrices générales sur l'utilisation des vaccins inactivés et des vaccins vivants atténués au sein de cette population, le CCNI estime que les avantages d'envisager, au cas par cas, l'administration du VRZ (plutôt que le WVCZ) chez ces personnes sont plus importants que les risques potentiels à l'heure actuelle. Le CCNI surveillera les données probantes en évolution et réévaluera la force de ses recommandations (à l'échelle de la santé publique comme à l'échelle individuelle) portant sur différentes populations et les personnes immunodéprimées de 18 ans et plus et ce, dès que les données probantes issues des essais en cours seront disponibles.

Le tableau 1 présente un résumé des recommandations mises à jour du CCNI sur I'utilisation du VVVCZ et du VRZ à I'échelle de la santé publique. Ces recommandations visent à appuyer les responsables provinciaux et territoriaux dans la prise de décisions relatives aux programmes de vaccination financés publiquement. La force de chaque recommandation ainsi que la qualité des données probantes sur lesquelles elle s'appuie sont indiquées.

Le tableau 2 présente un résumé des recommandations mises à jour du CCNI sur l'utilisation du VWVCZ et du VRZ à l'échelle individuelle. Ces recommandations s'adressent aux personnes qui souhaitent prévenir le zona ou aux cliniciens qui désirent conseiller leurs patients au sujet de la prévention du zona au moyen de vaccins qui ne sont présentement pas offerts par les programmes de vaccination de santé publique. La force de chaque recommandation ainsi que l'évaluation de la qualité des données probantes sur lesquelles elles s'appuient sont incluses.

Tableau 1 : Résumé des recommandations 2018 sur l'utilisation des vaccins contre le zona visant à appuyer la prise de décisions à l'échelle des programmes de santé publique ${ }^{a}$

\begin{tabular}{|c|c|c|}
\hline Type de vaccin & $\begin{array}{l}\text { Recommandation du CCNI } \\
\text { (Force de la recommandation) }\end{array}$ & $\begin{array}{l}\text { Qualité des données probantes appuyant la } \\
\text { recommandation }\end{array}$ \\
\hline \multirow[t]{5}{*}{ VRZ } & $\begin{array}{l}\text { 1. Le CCNI recommande d'offrir le VRZ aux personnes de } 50 \text { ans } \\
\text { et plus qui ne présentent pas de contre-indication. } \\
\text { (Forte recommandation du CCNI) }\end{array}$ & $\begin{array}{l}\text { Le CCNI conclut qu'il existe de bonnes données probantes pour } \\
\text { recommander une immunisation } \\
\text { (Données probantes de catégorie A) }\end{array}$ \\
\hline & $\begin{array}{l}\text { 2. Le CCNI recommande d'offrir le VRZ aux personnes de } 50 \text { ans } \\
\text { et plus qui ont déjà reçu le VVVCZ et qui ne présentent pas de } \\
\text { contre-indication. } \\
\text { (Forte recommandation du CCNI) }\end{array}$ & $\begin{array}{l}\text { Le CCNI conclut qu'il existe de bonnes données probantes pour } \\
\text { recommander une immunisation } \\
\text { (Données probantes de catégorie A) }\end{array}$ \\
\hline & $\begin{array}{l}\text { 2a. Chez les adultes de } 50 \text { ans et plus qui ont déjà reçu le VVVCZ, } \\
\text { le CCNI recommande d'envisager une revaccination par deux } \\
\text { doses du VRZ au moins un an après l'administration du VVVCZ. } \\
\text { (Recommandation discrétionnaire du CCNI fondée sur l'opinion } \\
\text { d'experts) }\end{array}$ & $\begin{array}{l}\text { Le CCNI conclut que les données probantes sont insuffisantes pour } \\
\text { formuler une recommandation sur l'intervalle entre l'administration du } \\
\text { VWVCZ et celle du VRZ. } \\
\text { (Données probantes de catégorie I) }\end{array}$ \\
\hline & $\begin{array}{l}\text { 3. Le CCNI recommande d'offrir le VRZ aux personnes de } 50 \text { ans } \\
\text { et plus qui ne présentent pas de contre-indication et qui ont des } \\
\text { antécédents de zona. } \\
\text { (Forte recommandation du CCNI) }\end{array}$ & $\begin{array}{l}\text { Le CCNI conclut qu'il existe des données probantes acceptables pour } \\
\text { recommander l'administration du vaccin. } \\
\text { (Données probantes de catégorie B) }\end{array}$ \\
\hline & $\begin{array}{l}\text { 3a. Chez les adultes de } 50 \text { ans et plus qui ont des antécédents } \\
\text { de zona, le CCNI recommande d'envisager une revaccination par } \\
\text { deux doses du VRZ au moins un an aprés l'épisode de zona. } \\
\text { (Recommandation discrétionnaire du CCNI fondée sur l'opinion } \\
\text { d'experts) }\end{array}$ & $\begin{array}{l}\text { Le CCNI conclut que les données probantes sont insuffisantes pour } \\
\text { formuler une recommandation sur l'intervalle entre l'épisode de zona } \\
\text { et l'administration du VRZ. } \\
\text { (Données probantes de catégorie I) }\end{array}$ \\
\hline WVCZ & $\begin{array}{l}\text { 4. Le CCNI recommande d'envisager l'administration du VVVCZ } \\
\text { aux personnes de } 50 \text { ans et plus qui sont immunocompétentes et } \\
\text { qui ne présentent pas de contre-indication, dans les cas où le VRZ } \\
\text { est contre-indiqué, s'il est impossible de l'obtenir ou lorsqu'il est } \\
\text { trop cher. } \\
\text { (Recommandation discrétionnaire du CCNI) }\end{array}$ & $\begin{array}{l}\text { Le CCNI conclut qu'il existe de bonnes données probantes pour } \\
\text { recommander l'administration du vaccin. } \\
\text { (Données probantes de catégorie A) }\end{array}$ \\
\hline $\begin{array}{l}\text { VRZ et VVVCZ } \\
\text { dans les } \\
\text { populations } \\
\text { immunodéprimées }\end{array}$ & $\begin{array}{l}\text { 5. Le CCNI recommande d'envisager, au cas par cas, } \\
\text { l'administration du VRZ (et non du VVVCZZ) chez les adultes } \\
\text { immunodéprimés de } 50 \text { ans et plus. } \\
\text { (Recommandation discrétionnaire du CCNI fondée sur l'opinion } \\
\text { d'experts) } \\
\text { Le CCNI passera en revue les données probantes en évolution et } \\
\text { réévaluera la force des recommandations. }\end{array}$ & $\begin{array}{l}\text { Le CCNI conclut que les données probantes sont insuffisantes à } \\
\text { I'heure actuelle pour recommander l'administration du vaccin. } \\
\text { (Données probantes de catégorie I) }\end{array}$ \\
\hline
\end{tabular}

Abréviations : CCNI, Comité consultatif national de l'immunisation; VRZ, vaccin sous-unitaire recombinant; VVVZ, vaccin à virus vivant atténué contre le zona

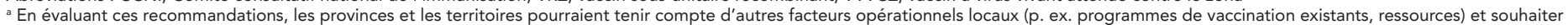

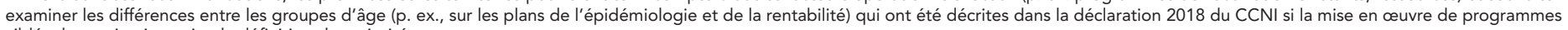
ciblés de vaccination exige la définition des priorités 
Tableau 2 : Résumé des recommandations 2018 sur l'utilisation des vaccins contre le zona visant à appuyer la prise de décisions à l'échelle individuelle ${ }^{a}$

\begin{tabular}{|c|c|c|}
\hline Type de vaccin & $\begin{array}{l}\text { Recommandation du CCNI } \\
\text { (Force de la recommandation) }\end{array}$ & $\begin{array}{l}\text { Qualité des données probantes appuyant } \\
\text { la recommandation }\end{array}$ \\
\hline \multirow[t]{5}{*}{ VRZ } & $\begin{array}{l}\text { 1. Le CCNI recommande d'offrir le VRZ aux personnes de } 50 \text { ans et } \\
\text { plus qui ne présentent pas de contre-indication. } \\
\text { (Forte recommandation du CCNI) }\end{array}$ & $\begin{array}{l}\text { Le CCNI conclut qu'il existe de bonnes données } \\
\text { probantes pour recommander une immunisation } \\
\text { (Données probantes de catégorie A) }\end{array}$ \\
\hline & $\begin{array}{l}\text { 2. Le CCNI recommande d'offrir le VRZ aux personnes de } 50 \text { ans et } \\
\text { plus qui ont déjà reçu le VVVCZ et qui ne présentent pas de contre- } \\
\text { indication. } \\
\text { (Forte recommandation du CCNI) }\end{array}$ & $\begin{array}{l}\text { Le CCNI conclut qu'il existe de bonnes données } \\
\text { probantes pour recommander une immunisation } \\
\text { (Données probantes de catégorie A) }\end{array}$ \\
\hline & $\begin{array}{l}\text { 2a. Chez les adultes de } 50 \text { ans et plus qui ont déjà reçu le VVVCZ, le } \\
\text { CCNI recommande d'envisager une revaccination par deux doses du } \\
\text { VRZ au moins un an après l'administration du WVVCZ. } \\
\text { (Recommandation discrétionnaire du CCNI fondée sur l'opinion } \\
\text { d'experts) }\end{array}$ & $\begin{array}{l}\text { Le CCNI conclut que les données probantes sont } \\
\text { insuffisantes pour formuler une recommandation sur } \\
\text { l'intervalle entre l'administration du VVVCZ et celle du } \\
\text { VRZ. } \\
\text { (Données probantes de catégorie I) }\end{array}$ \\
\hline & $\begin{array}{l}\text { 3. Le CCNI recommande d'offrir le VRZ aux personnes de } 50 \text { ans } \\
\text { et plus qui ne présentent pas de contre-indication et qui ont des } \\
\text { antécédents de zona. } \\
\text { (Forte recommandation du CCNI) }\end{array}$ & $\begin{array}{l}\text { Le CCNI conclut qu'il existe des données probantes } \\
\text { acceptables pour recommander l'administration du } \\
\text { vaccin. } \\
\text { (Données probantes de catégorie B) }\end{array}$ \\
\hline & $\begin{array}{l}\text { 3a. Chez les adultes de } 50 \text { ans et plus qui ont des antécédents de } \\
\text { zona, le CCNl recommande d'envisager une revaccination par deux } \\
\text { doses du VRZ au moins un an après l'épisode de zona. } \\
\text { (Recommandation discrétionnaire du CCNI fondée sur l'opinion } \\
\text { d'experts) }\end{array}$ & $\begin{array}{l}\text { Le CCNI conclut que les données probantes sont } \\
\text { insuffisantes pour formuler une recommandation sur } \\
\text { l'intervalle entre l'épisode de zona et l'administration } \\
\text { du VRZ. } \\
\text { (Données probantes de catégorie I) }\end{array}$ \\
\hline VVVCZ & $\begin{array}{l}\text { 4. Le CCNI recommande d'envisager l'administration du VVVCZ aux } \\
\text { personnes de } 50 \text { ans et plus qui sont immunocompétentes et qui } \\
\text { ne présentent pas de contre-indication, dans les cas où le VRZ est } \\
\text { contre-indiqué, s'il est impossible de l'obtenir ou lorsqu'il est trop } \\
\text { cher. } \\
\text { (Recommandation discrétionnaire du CCNI) }\end{array}$ & $\begin{array}{l}\text { Le CCNI conclut qu'il existe de bonnes données } \\
\text { probantes pour recommander l'administration du } \\
\text { vaccin. } \\
\text { (Données probantes de catégorie A) }\end{array}$ \\
\hline $\begin{array}{l}\text { VRZ et VVVCZ dans les } \\
\text { populations immunodéprimées }\end{array}$ & $\begin{array}{l}\text { 5. Le CCNI recommande d'envisager, au cas par cas, l'administration } \\
\text { du VRZ (et non du WVVCZ) chez les adultes immunodéprimés de } 50 \\
\text { ans et plus. } \\
\text { (Recommandation discrétionnaire du CCNI fondée sur l'opinion } \\
\text { d'experts) } \\
\text { Le CCNI passera en revue les données probantes en évolution et } \\
\text { réévaluera la force des recommandations. }\end{array}$ & $\begin{array}{l}\text { Le CCNI conclut que les données probantes sont } \\
\text { insuffisantes à l'heure actuelle pour recommander } \\
\text { I'administration du vaccin. } \\
\text { (Données probantes de catégorie I) }\end{array}$ \\
\hline
\end{tabular}

Abréviation : CCNI, Comité consultatif national de l'immunisation

a En évaluant ces recommandations, les personnes et les cliniciens pourraient souhaiter examiner les points de décision relatifs au vaccin et à l'âge au moment de la vaccination décrits dans la déclaration 2018 du CCNI

\section{Conclusion}

Le CCNI a conclu que le VRZ comme le WVCZ sont immunogènes, rentables et sûrs, ainsi qu'efficaces dans la réduction de l'incidence du zona et de la névralgie post-zostérienne. Cependant, l'efficacité du VVVCZ s'atténue avec l'âge d'administration et le temps écoulé depuis la vaccination, alors que celle du VRZ demeure élevée et semble diminuer plus lentement que celle du VVVCZ, et ce, dans tous les groupes d'âge. Dans les trois ans suivant la vaccination, l'efficacité du VRZ contre l'incidence du zona et de la névralgie post-zostérienne semble atteindre le double de celle du VVVCZ. L'efficacité du VRZ contre l'incidence du zona demeure constante dans les quatre ans suivant la vaccination et aucune diminution significative n'a été observée avec le temps. Par contre, une baisse significative de la protection offerte par le WVCZ a été observée un an après la vaccination. L'efficacité sur quatre ans du VRZ à l'égard de l'incidence du zona ne varie pas de façon significative entre les groupes d'âge; par contre, le VWVCZ est significativement moins efficace chez les adultes de plus de 70 ans que chez ceux de 50 à 59 ans. En raison de la présence de l'adjuvant, qui provoque une forte réponse immunitaire au niveau cellulaire aidant à compenser la baisse de l'immunité liée au vieillissement, le VRZ est plus réactogène que le VVVCZ. II s'agit toutefois d'une réactogénicité transitoire et l'éducation jouera un rôle crucial pour promouvoir l'adhésion à la deuxième dose du VRZ.
Comparativement à l'absence de vaccination, les deux vaccins sont rentables chez les personnes de 50 ans et plus, en particulier chez les 65 à 79 ans. En effet, le fardeau de la maladie augmente avec l'âge (en raison du risque accru d'hospitalisations et de névralgie post-zostérienne par cas de zona, surtout chez les 65 ans et plus) et la probabilité que le vaccin soit efficace durant les années où le fardeau de la maladie est le plus important améliore sa rentabilité, à moins que l'efficacité du vaccin ne baisse rapidement. De plus, les bienfaits de la vaccination s'accumulent sur une période prolongée, en raison de l'espérance de vie supérieure au sein de ce groupe d'âge, comparativement aux personnes de 80 ans et plus. Du point de vue de la santé publique, le vaccin contre le zona peut être administré en même temps que d'autres vaccins destinés aux adultes, de manière à améliorer la couverture vaccinale et à réduire les frais d'exécution. Le VRZ est plus rentable que le WVCZ, pour tous les groupes d'âge étudiés.

Selon les données probantes passées en revue, le CCNI recommande la vaccination contre le zona.

\section{Déclaration des auteurs}

R. W. - Rédaction de l'ébauche initiale du résumé - Révision et édition 
S. I. - Rédaction de l'ébauche initiale du résumé - Révision et édition

Ce résumé de la déclaration sur le zona du $\mathrm{CCNI}$ a été préparé par S. Ismail, M. Tunis, O. Baclic, K. Eng, M. K. Doll, M. J. Hu, $\mathrm{S}$. Duchesne-Belanger et R. Warrington, puis approuvé par le CCNI.

\section{Conflit d'intérêts}

Aucun.

\section{Remerciements}

Membres du groupe de travail sur le zona : R. Warrington (président), S. Deeks, P. De Wals, K. Dooling, K. Eng, J. Gallivan, S. Ismail, M. Landry, C. Rotstein, C. Sauvageau, S. E. Straus, M. Tunis

Membres du CCNI : C. Quach (présidente), W. Vaudry (vice-présidente), N. Dayneka, P. DeWals, S. Deeks, V. Dubey, R. Harrison, M. Lavoie, C. Rotstein, M. Salvadori, B. Sander, N. Sicard, R. Warrington

Représentants de liaison : J. Brophy (Association canadienne pour la recherche et l'évaluation en immunisation), E. Castillo (Société des obstétriciens et gynécologues du Canada), A. Cohn (Centers for Disease Control and Prevention, États-Unis), T. Cole (Comité canadien sur l'immunisation), J. Emili (Collège des médecins de famille du Canada), K. Klein (Conseil des médecins hygiénistes en chef), C. Mah (Association canadienne de santé publique), D. Moore (Société canadienne de pédiatrie), A. Pham-Huy (Association pour la microbiologie médicale et I'infectiologie Canada)

Représentants d'office : K. Barnes (ministère de la Défense nationale et les Forces armées canadiennes), G. Charos (Centre de l'immunisation et des maladies respiratoires infectieuses [CIMRI], Agence de la santé publique du Canada [ASPC]), $\mathrm{G}$. Coleman (Direction des produits biologiques et des thérapies génétiques [DPBTG], Santé Canada [SC]), J. Gallivan (Direction des produits de santé commercialisés [DPSC], SC), J. Pennock (CIMRI, ASPC) M. G. Poliquin (Laboratoire national de microbiologie) et T. Wong (Direction générale de la santé des Premières nations et des Inuits [DGSPNI], SC)

Le CCNI souhaite remercier les groupes suivants pour leur contribution : Les membres de l'équipe de I'Université Laval, du Centre de recherché du CHU de Québec et de l'INSPQ, notamment M. Brisson, Z. Zhou, M. Drolet, C. Sauvageau, P. De Wals, V. Gilca, M. J.-F. Laprise et R. Amini pour leur contribution à l'analyse économique. Cette équipe n'a cependant pas rédigé la section économique de la présente déclaration. L. Gamble (Biblitothèque de la santé, SC) pour avoir soutenu la stratégie de recherche sur l'immunogénicité, ainsi que les membres de l'équipe MAGIC, notamment A. C. Tricco, S. E. Straus, W. Zarin, R. Cardoso, A. A. Veroniki, M. P. A. Khan, V. Nincic, M. M. Ghassemi, R. Warren, J. Sharpe et A. Page pour leur contribution à l'analyse de l'innocuité et de l'efficacité. La stratégie de recherche dans la littérature sur l'innocuité et l'efficacité a été mise au point et revue par des pairs par les libraires E. Cogo et J. McGowan

\section{Financement}

Les travaux du CCNI sont financés par l'Agence de la santé publique du Canada.

\section{Références}

1. Brisson M, Edmunds WJ, Law B, Gay NJ, Walld R, Brownell M, Roos LL, De Serres G. Epidemiology of varicella zoster virus infection in Canada and the United Kingdom. Epidemiol Infect 2001 Oct;127(2):305-14. https://doi.org/10.1017/ S0950268801005921. PubMed (https://www.ncbi.nlm.nih. gov/entrez/query.fcgi?cmd=Retrieve\&db=PubMed\&list_ uids $=11693508 \&$ dopt $=$ Abstract)

2. Kawai K, Gebremeskel BG, Acosta CJ. Systematic review of incidence and complications of herpes zoster: towards a global perspective. BMJ Open 2014 Jun;4(6):e004833. https://doi. org/10.1136/bmjopen-2014-004833. PubMed (https://www. ncbi.nlm.nih.gov/entrez/query.fcgi? cmd=Retrieve $\& d b=P u b M e$ d\&list_uids=24916088\&dopt=Abstract)

3. Pinchinat $\mathrm{S}$, Cebrián-Cuenca AM, Bricout $H$, Johnson RW. Similar herpes zoster incidence across Europe: results from a systematic literature review. BMC Infect Dis 2013 Apr;13:170. https://doi.org/10.1186/1471-2334-13-170. PubMed (https:// www.ncbi.nlm.nih.gov/entrez/query.fcgi?cmd=Retrieve\&db=Pu bMed\&list_uids=23574765\&dopt=Abstract)

4. Marra F, Chong M, Najafzadeh M. Increasing incidence associated with herpes zoster infection in British Columbia, Canada. BMC Infect Dis 2016 Oct;16(1):589. https://doi. org/10.1186/s12879-016-1898-z. PubMed (https://www.ncbi. $\mathrm{nlm}$.nih.gov/entrez/query.fcgi?cmd=Retrieve\& $\mathrm{db}=$ PubMed\&li st_uids=27765026\&dopt=Abstract)

5. Russell ML, Dover DC, Simmonds KA, Svenson LW. Shingles in Alberta: before and after publicly funded varicella vaccination. Vaccine 2014 Oct;32(47):6319-24. https://doi.org/10.1016/j. vaccine.2013.09.018. PubMed (https://www.ncbi.nlm.nih. gov/entrez/query.fcgi?cmd=Retrieve\&db=PubMed\&list_ uids $=24099868 \&$ dopt $=$ Abstract )

6. Russell ML, Schopflocher DP, Svenson L, Virani SN. Secular trends in the epidemiology of shingles in Alberta. Epidemiol Infect 2007 Aug;135(6):908-13. https://doi.org/10.1017/ S0950268807007893. PubMed (https://www.ncbi.nlm.nih. gov/entrez/query.fcgi?cmd=Retrieve\&db=PubMed\&list_ uids=17291380\&dopt=Abstract)

7. Tanuseputro P, Zagorski B, Chan KJ, Kwong JC. Population-based incidence of herpes zoster after introduction of a publicly funded varicella vaccination program. Vaccine 2011 Nov;29(47):8580-4. https://doi.org/10.1016/j. vaccine.2011.09.024. PubMed (https://www.ncbi.nlm.nih. gov/entrez/query.fcgi?cmd=Retrieve\&db=PubMed\&list_ uids=21939721\&dopt=Abstract)

8. Edgar BL, Galanis E, Kay C, Skowronski D, Naus M, Patrick D. The burden of varicella and zoster in British Columbia 19942003: baseline assessment prior to universal vaccination. Can Commun Dis Rep 2007 Nov;33(11):1-15. PubMed (https:// www.ncbi.nlm.nih.gov/entrez/query.fcgi?cmd=Retrieve\&db=Pu bMed\&list_uids=18163240\&dopt=Abstract)

9. Ouhoummane N, Boulianne N, De Serres G, De Wals P, Brisson M. Fardeau de la varicelle et du zona au Québec, 1990-2008: impact du programme universel de vaccination. Québec (QC): Institut National de Santé Publique du Québec; 2011. www. 
inspq.qc.ca/pdf/publications/1355_FardeauVaricelleZona19002008ImpactUnivVaccin.pdf

10. Comité consultatif national de l'immunisation (CCNI). Déclaration sur I'utilisation recommandée du vaccin contre le virus de I'herpès zoster. Relevé des maladies transmissibles au Canada 2010; 36 (ACS-1). https://doi.org/10.14745/ccdr. v36i00a01

11. Comité consultatif national de l'immunisation (CCNI). Mise à jour de l'utilisation du vaccin contre le zona. Agence de la santé publique du Canada, Février 2014. Consulté en Avril 2018. https://www.canada.ca/fr/sante-publique/services/ publications/vie-saine/mise-a-jour-utilisation-vaccin-contrezona.html

12. Canadian Immunization Committee $(\mathrm{ClC})$. Recommendations for Zoster Immunization Programs. Ottawa (ON): PHAC; 2014 http://publications.gc.ca/collections/collection_2014/aspcphac/HP40-106-2014-eng.pdf

13. Ministère de la santé et des soins de longue durée de I'Ontario. Programme public d'immunisation contre le zona:
Information destinée aux professionnels de la santé. Ottawa (ON): MSSLD; 2016. http://www.health.gov.on.ca/fr/pro/ programs/immunization/docs/shingles_hcp_qa_fr.pdf

14. Comité consultatif national de l'immunisation (CCNI). Mise à jour de l'utilisation du vaccin contre le zona. Ottawa (ON): ASPC; 2018. https://www.canada.ca/fr/services/sante/ publications/vie-saine/recommandations-jour-utilisationvaccins-contre-zona.html

15. Comité consultatif national de l'immunisation (CCNI). Recommandations pour l'immunisation fondées sur des données probantes - Méthodes du Comité consultatif national de l'immunisation. RMTC. 2009;35(ACS-1):1-10. PubMed (https://www.ncbi.nlm.nih.gov/entrez/query.fcgi?cmd=Retrieve $\& d b=$ PubMed\&list_uids=19192504\&dopt=Abstract)

16. Comité consultatif national de l'immunisation (CCNI). Vaccin contre le zona: Guide d'immunisation canadien. Ottawa (ON): PHAC; Mis à jour 2018. www.canada.ca/en/public-health/ services/publications/healthy-living/canadian-immunizationguide-part-4-active-vaccines/page-8-herpes-zoster-(shingles)vaccine.html 